\title{
On the requirements to establish a European radiological preparedness for malicious airborne dispersion scenarios
}

\author{
K.G. Andersson ${ }^{1}$, P. Astrup ${ }^{1}$, P. Roos ${ }^{1}$, T. Mikkelsen ${ }^{1}$, S.C. Hoe ${ }^{2}$, \\ L.H. Jacobsen ${ }^{3}$, L. Schou-Jensen ${ }^{3}$, J. Pehrsson ${ }^{3}$ and S.P. Nielsen ${ }^{1}$ \\ ${ }^{1}$ Risø National Laboratory for Sustainable Energy, Technical University of Denmark, \\ P.O. Box 49, DK-4000 Roskilde, Denmark \\ ${ }^{2}$ Danish Emergency Management Agency, Datavej 16, DK-3460 Birkerød, Denmark \\ ${ }^{3}$ Prolog Development Center, HJ Holst Vej 3C-5C, DK-2605 Brøndby, Denmark
}

\begin{abstract}
European computerised decision support systems are currently targeted for large accidental atmospheric contaminant releases from nuclear installations. To make these systems applicable also for malicious dispersion events, such as 'dirty bomb' blasts, a series of modifications and extensions are necessary. Also European decision support handbooks need supplementary sections to cover the features of these types of scenarios. An overview is given of the requirements.
\end{abstract}

\section{INTRODUCTION}

Current European capabilities to effectively address emergency situations resulting from terror attacks involving atmospheric dispersion of radioactive matter are generally very modest. A radiological dispersion device such as a 'dirty bomb' can lead to contamination of much of a large city over a period of minutes, and to avoid long lasting mass disruption and infrastructural breakdown with immense repercussions, it is essential to have an operational preparedness with reliable supportive tools available from the very start. In national European emergency plans, where available for such incidents, there is a tendency to focus on the very early phase, where people may become injured by the blast. However, the complex and potentially highly time-consuming tasks of cleaning a large city area, which may have been contaminated by a single radionuclide that is difficult to detect and map (e.g., a pure alpha emitter), are rarely seen to have been given much thought. Here decision support systems enabling a prognostic overview of the contamination and its consequences on the basis of available observations can play a particularly important role. Such systems are also needed in any intervention optimisation, to predict averted and residual doses [1]. The ARGOS and RODOS decision support systems have been developed to facilitate effective European emergency management and are used by authorities and their advisors in most European countries. Unlike other common systems on the market (some of which must be considered obsolete in view of the current knowledge), these are targeted to reflect European conditions and can readily be modified as needed. However, these systems were developed for decision support in connection with accidental releases from nuclear installations, and can for a number of reasons, in their present form, not be applied for terror-related scenarios like 'dirty bombs'. This paper is aimed at providing an overview of important shortcomings in this context. Also the new European handbook for assisting in the management of radiologically contaminated inhabited areas, which is being integrated in the preparedness in many European countries, urgently needs supplementary information to make it applicable for terror scenarios, and also these needs are briefly discussed in the paper. 


\section{METHODS AND RESULTS}

\subsection{European decision support systems}

European computerised decision support systems (essentially models for estimation of doses that would be received under different circumstances due to an airborne contaminant release) need to enable adequately detailed and reliable modelling of the physical and chemical processes governing dose to humans in the given scenario. Malicious airborne dispersion could occur in a number of different types of scenarios, which would all involve plume propagation at a much lower altitude than implicitly assumed in the original ARGOS and RODOS systems. In principle, probably the simplest way of generating a haze of small radioactive particles would be to continuously operate some sort of nebuliser arrangement, e.g., from a rooftop. However, to inflict real harm on an exposed population, the source would need to have considerable strength, wherefore its placement and set-up would be far from trivial, and the release height might well still not be sufficient to cause widespread contamination over a city area. An alternative that could cause very widespread airborne contamination would be dispersion from an aircraft. However, with the recent tightening of security in air traffic, and considering the weight and volume of shielding that would be required to enable transportation of a strong source without almost instantly killing the terrorist, this would be a very highly challenging, if not impossible, task. For instance, a $1000 \mathrm{TBq}{ }^{60} \mathrm{Co}$ teletherapy source, which might possibly be orphaned or stolen, would, if handled without shielding, give a lethal dose within seconds. Pure beta emitters like ${ }^{90} \mathrm{Sr}$ would be somewhat easier to shield, but e.g., the Bremsstrahlung problem would still be highly problematic at this source strength level. A means for airborne contaminant dispersion that has attracted particularly great attention, also due to its dramatic nature, which might be appealing to a terrorist, is the 'dirty bomb'. The presentation in this paper will focus on this latter type of dispersion device. It has been speculated that a strong radioactive source with sufficient shielding might be transported into position in a city area on a lorry or other vehicle. It might however well be a challenge to effectively detonate a bomb from a bulky shielding arrangement so that it leads to atmospheric dispersion of small particles. It should be noted that although experiments have been made in various countries mimicking the detonation of a 'dirty bomb' using, e.g., non-radioactive rare earth tracers, short-lived radionuclides such as ${ }^{99 \mathrm{~m}} \mathrm{Tc}$, or coloured powder that could visibly be followed in the wind [2-4], none of these have considered or even noted the possible difficulties in having to carry out the explosion from a shielding arrangement.

\subsubsection{Atmospheric plume dispersion}

In modelling the dispersion and subsequent deposition on different surfaces in an inhabited environment (e.g., soil, grass, plants, pavements, streets, exterior building walls, roofs, indoor surfaces, food products, human lungs, skin, hair, clothing) of aerosolised radionuclides after the detonation of a 'dirty bomb', it is essential to first determine the initial elevation height distribution and width of the plume containing the contaminant aerosols generated by the blast. This is essential information required by atmospheric dispersion models (at any resolution scale) to estimate how the contamination will in time spread out over an inhabited area. The initial rise of the contaminated material after a 'dirty bomb' blast will occur due to buoyancy and initial momentum. As the cloud rises, its movement will cause turbulent mixing with non-buoyant ambient air. Deceleration through decreasing buoyancy will reduce the boundary turbulence to that of the ambient air, and at that point, a cloud shape will have formed that could be regarded as the 'initial' spatial distribution before ambient wind driven dispersion takes over. Already in the early cold war period, Morton et al. [5] demonstrated theoretically that the initial cloud top height, $\mathrm{H}$, to which the cloud from an explosion rises, can be described by an equation of the type $\mathrm{H}=\mathrm{a} \cdot(\mathrm{b} / \mathrm{c})^{1 / 4}$, where $\mathrm{a}$ is a constant, whereas $\mathrm{b}$ and $\mathrm{c}$ represent respectively the initial buoyant force and ambient stability. They also parameterised the equation according to an explosion caused by detonation 
of TNT (trinitrotoluene), and demonstrated that the term b/c in the above equation was proportional to the mass of TNT applied. Empirical relationships of this type have since then been suggested by other workers on the basis of experimentation in open fields [4, 6]. These have also suggested explosive mass based formulae for the cloud's 'cap' (i.e. top part) radius. There is some discrepancy regarding reported values of particularly the constant $\mathrm{a}$ in the above equation, probably relating to different geometrical bomb constructions and possibly differences in stability. It should be noted that this formula has only been proved valid for explosions in open areas. None of the existing European decision support systems can apply this type of plume information for conventional explosions. Therefore, an initial plume shape model has been suggested, partially on the basis of classified information, for application in the ARGOS decision support system, which also takes into account the relative distribution of the contaminant mass in different parts of the 'cap' and 'stem' of the 'initial' cloud. The later dispersion of different fractions of the plume can then be modelled in ARGOS by separate puffs.

The plume originating from a 'dirty bomb' would be likely to at least partially be dispersed at such low altitude that interactions with obstacles like buildings and trees could have an important bearing on the dispersion and deposition pattern. Parts of the plume can be entrained and delayed behind obstacles in the landscape, which adds to the complexity of the spatial contaminant distribution. The introduction of a recently created high-resolution dispersion model, URD, in ARGOS has enabled modelling of such effects, following the trajectories of both primary puffs and secondary puff sections representing different parts of the contaminated air mass. The URD model also takes into account the increased small scale turbulence and limitations on horizontal eddy movements due to the comparatively low plume altitude. As has been demonstrated [7, 8], these factors can affect the contamination pattern, wherefore the original larger scale dispersion models in ARGOS and RODOS are not well suited for 'dirty bomb' scenario estimation. It should be noted that ARGOS and RODOS are currently not equipped with models that can predict the airborne dispersion of contaminants in large indoor and semi-confined spaces like underground train stations and airports.

\subsubsection{Contaminant deposition}

Contaminant deposition will be strongly influenced by precipitation during the plume passage, as plume rainout and washout are powerful deposition processes. Both dry and wet deposition will very much depend on particle size (see, e.g. [9]). This type of dependencies have only recently been considered in the ARGOS and RODOS decision support systems, where a library of deposition velocities [10] has been introduced to distinguish between the small (slightly less than one micron) particles likely to be formed in a large nuclear power plant accident by volatile radiocontaminants like caesium, tellurium and molybdenum and the somewhat larger (typically 2-4 $\mu \mathrm{m}$ ) particles of more refractory contaminants (e.g., strontium, zirconium and cerium) that were for instance observed at considerable distance after the Chernobyl accident. However, the size spectra of particles that could contaminate an inhabited area in a 'dirty bomb' scenario are much more complex, and here it is essential to distinguish between different types of particle formation processes. As explained by Andersson et al. [8] on the background of the experimentation of Harper et al. [2], particle size distributions produced by 'dirty bombs' depend on elemental properties of the contaminants, as well as their physicochemical forms and the construction of the bomb. Some types of sources can under sufficient pressure from the explosion effectively undergo shock aerosolisation, thus forming small particles that may be transported over considerable distances in a city. The smallest particles are formed after phase transition (either to vapour or liquid), but phase transition is not possible for all contaminant elements and initial physicochemical forms (e.g., ceramics, powders/salts, metals, liquids). Metals like cobalt are for instance exceedingly difficult to aerosolise, and the aerosol size spectra from competent bomb explosions with ceramics and liquids will be very different, since aerosols formed from ceramic sources are generally the result of physical fractionation rather than phase transition. Whereas a liquid source bomb detonation may produce large amounts of 
submicron particles with low deposition velocity (thus having potential to disperse over a very large area), much of the aerosol produced in a bomb detonation involving a ceramic contaminant will have sizes exceeding $10 \mu \mathrm{m}$, and thus due to gravitational settling have dry deposition velocities exceeding that of a $0.5 \mu \mathrm{m}$ particle by orders of magnitude. If these differences are not taken into account in a decision support model, both absolute contamination level and dose estimates and the size and location of the contaminated areas will be completely wrongly predicted. Decision support models are therefore required to distinguish between a limited number of aerosolisation processes and contain adequate representation of the deposition process, and this is currently not the case for the European decision support systems.

It should be noted that also deposition on humans could be particularly important in 'dirty bomb' scenarios involving larger particles, which could give a comparatively high dose before they are cleared off. State-of-the-art knowledge on aerosol contamination of humans [11] is so far not integrated in the European decision support systems, and also here, aerosol size is of key importance.

\subsubsection{Natural and forced removal of deposited contaminants}

In modelling doses that would be received over time with and without intervention, it is essential to estimate the natural migration and weathering of contaminants deposited on the different surfaces in the environment. A problem is here that the parameters governing the estimates of natural migration of contaminants in ARGOS and RODOS are practically exclusively based on measurements of the behaviour of Chernobyl radiocaesium (i.e. caesium in readily soluble form). The caesium cation has the feature that it binds very strongly and selectively in minerals that are highly abundant in most common construction materials, as well as in soil and street dust [12]. As the Chernobyl accident demonstrated, radiocaesium contamination levels on construction materials only very slowly declined (often with a half-life of many years), whereas contaminants embedded in larger and less soluble particles were much easier removed from impermeable surfaces near the Chernobyl NPP. Literally no other perceivable contaminant ion would be nearly as strongly fixed on urban surfaces and in soil as the caesium cation. This may not be of critical importance in connection with modelling of the long-term radiological consequences of large nuclear power plant accidents, where radiocaesium would anyway play a dominant role, but in connection with a 'dirty bomb' dispersion scenario, which could involve a single and very different contaminant, long term doses could be overestimated by many orders of magnitude if the caesium cation weathering parameters, which are currently the only available ones in the ARGOS and RODOS systems, are applied. Physicochemical forms of the contaminants could and should be taken into account here. Obviously also the efficiency factors for countermeasures involving contaminant removal, which are in the European decision support systems also based on Chernobyl experience, should be re-evaluated to provide reliable estimates for other contaminants than caesium. It would also be worth considering if other countermeasures than those traditionally suggested for nuclear emergencies might be better suited for some of the contaminants that would be of greatest concern in connection with 'dirty bomb' scenarios.

\subsubsection{Other important factors}

Malicious dispersion scenarios could involve a number of radionuclides that are not traditionally considered in nuclear power plant preparedness and thus not presently included in ARGOS and RODOS. Dose rate conversion factors (i.e. factors giving the dose rate in a given receptor geometry per unit contamination on/in a given surface in the environment) need to be calculated and included for these radionuclides, some of which are pure beta emitters.

Air concentrations of contaminants ingressed into buildings after an outdoor explosion will depend on ventilation rates, indoor deposition rates and filtration of air entering the building canopy. Both of the latter two of these parameters have a strong dependence on particle size. For the particle sizes currently 
featured in the European decision support systems there is practically no building filtration, whereas with respect to the $10 \mu \mathrm{m}$ particle size that would be highly relevant to some conceivable 'dirty bomb' scenarios, building filtration would typically remove about $90 \%$ of the particles from the air entering the building [13]. Also the indoor deposition of $10 \mu \mathrm{m}$ particles would be about an order of magnitude higher than that of particles in the $1 \mu \mathrm{m}$ range. If this problem is not taken into account, calculated indoor air contamination level estimates will obviously be misguiding.

It should also be mentioned that the generally applied inhalation dose conversion factors (from ICRP) based on a rough solubility class categorisation could be improved substantially by taking into account actual physicochemical forms of strong sources of concern. Finally, it should be noted that ingestion of a hot meal to which deposition of large contaminant particles has occurred can lead to a significant dose [8], demanding attention.

\subsection{European handbooks}

The Commission of the European Communities has recently released a CD containing a suite of three handbooks [14] to assist in the management of contaminated inhabited areas, food production systems and drinking water following a nuclear or radiological emergency. The handbooks have been developed in collaboration with a wide range of stakeholder organisations on different levels, and include detailed descriptions in a standardised datasheet format of countermeasures, which may be implemented in different time phases following an emergency. Although the focus is on releases following large nuclear power plant accidents, the three handbooks contain much generic information that can also be valuable in connection with other types of contaminant release incident scenarios. Particularly the handbook dealing with contamination in inhabited areas [15] has relevance in relation to 'dirty bomb' scenarios, as such attacks would be expected to occur in a city. Among the features of this handbook are, besides the countermeasure datasheets, a framework of selection tables, decision trees and worked examples designed to aid in the decision process, supporting information and analyses, and sections on societal and ethical issues as well as communication and information needs. The handbooks are currently being integrated in the preparedness in a number of European countries, both for training, exercises, preplanning purposes and as an information resource in the event of an emergency. However, to fully realise the potential of the handbooks in relation to malicious dispersion scenarios, rather comprehensive supplementary sections are needed to describe those aspects of a malicious dispersion scenario, which require special handling. For instance, as mentioned above, special countermeasures might be devised, which would be more suitable for some malicious dispersion scenarios than those currently described in the handbook datasheets, and the present descriptions should be revised or supplemented with respect to targeted radionuclides (e.g., since the contaminant could be a single pure alpha or beta emitter), scale of application (which would be comparatively limited for a 'dirty bomb' scenario), and time scale (since the immediate emergency phase in a 'dirty bomb' scenario would be very short). Also for instance guidance sections on implications for recovery of timing, size of the affected area, relative importance of cleaning surfaces, discussions of likely contaminants, their environmental mobility and resulting doses, monitoring and measurement requirements, societal and communication aspects, and countermeasure elimination criteria need supplements.

\section{CONCLUSIONS}

European computerised decision support systems for nuclear emergencies have many useful computation features, user interfaces and graphic representation possibilities that would be invaluable in the establishment of a state-of-the-art European based consequence estimation tool for malicious contaminant dispersion scenarios. However, it should be stressed that model and parameter refinements are urgently needed to provide reliable consequence estimation for this particular category of scenarios. Important current shortcomings relate to, e.g., the representation of atmospheric dispersion, contaminant 
deposition, and natural and forced removal of deposited contaminants. Improvements could and should be made, to a great extent based on existing knowledge of materials and processes. Also the European handbook for managing contaminated inhabited areas needs supplementary sections for malicious contaminant dispersion scenarios.

\section{References}

[1] ICRP, Publication 103, Annals of the ICRP, (ICRP, Vienna, Austria, 2007).

[2] F.T. Harper, S.V. Musolino, W.B. Wente, Health Phys. 93(1), 1 (2007).

[3] Z. Prouza, V. Beckova, I. Cespirova, J. Helebrant, J. Hulka, P. Kuca, V. Michalek, P. Rulik, J. Skrkal, J. Hovorka, Rad. Prot. Dosimetry 139(4), 519 (2010).

[4] H. Thielen, E. Schrödl, Blast experiments for the derivation of initial cloud dimensions after a 'Dirty Bomb' event, presented at EUROSAFE 2004 Forum for Nuclear Safety, Berlin, Germany, 2004.

[5] B.R. Morton, G. Taylor, J.S. Turner, Proc. Roy. Soc. A, V234, 1 (1956).

[6] V.P. Reshetin, J.L. Regens, Modeling Cs-137 dispersion from a radiological dispersion device, presented at International Conference on Technical Means for Prevention of Radiation Terrorism and Liquidation of its Consequences, St. Petersburg, Russia, 2004.

[7] P. Astrup, S. Thykier-Nielsen, T. Mikkelsen, Ris $\varnothing-R-1539(E N)$, Ris $\varnothing$, Roskilde, Denmark, 2005.

[8] K.G. Andersson, T. Mikkelsen, P. Astrup, S. Thykier-Nielsen, L.H. Jacobsen, S.C. Hoe, S.P. Nielsen, J. Environ. Radioactivity 100, 1005 (2009).

[9] S.P. Nielsen, K.G. Andersson (eds.), NKS report NKS-232, Nordic Nuclear Safety Research (NKS), Roskilde, Denmark, 2011.

[10] T.W. Charnock, J.A. Jones, L.N. Singer, K.G. Andersson, J. Roed, S. Thykier-Nielsen, T. Mikkelsen, P. Astrup, J.C. Kaiser, H. Müller, G. Pröhl, W. Raskob, S.C. Hoe, L.H. Jacobsen, L. Schou Jensen, F. Gering, Radioprotection 44(5), 407 (2009).

[11] K.G. Andersson, C.L. Fogh, M.A. Byrne, J. Roed, A.J.H. Goddard, S.A.M. Hotchkiss, Health Phys. 82(2), 226 (2002).

[12] K.G. Andersson, in Airborne radioactive contamination in inhabited areas: Radioactivity in the Environment vol. 15, edited by K.G. Andersson (Elsevier, Amsterdam, Netherlands, 2009), 107.

[13] C.M. Long, H.H. Suh, P.J. Catalano, P. Koutrakis, P., Environ. Sci. Technol. 35(10), 2089 (2001).

[14] A.F. Nisbet, J. Brown, B.J. Howard, N.A. Beresford, H. Ollagnon, C. Turcanu, J. Camps, K. Andersson, A. Rantavaara, T. Ikäheimonen, T. Duranova, D. Oughton, G. Kirchner, C. Papachristodoulou, K. Ioannides, P. Kwakman, Radioprotection vol. 45(5), S23 (2010).

[15] A.F. Nisbet, J. Brown, H. Rochford, T. Cabianca, A. Jones, K. Andersson, T. Duranova, R. Hänninen, T. Ikäheimonen, G. Kirchner, V. Bertsch, F. Gallay, N. Reales, Generic handbook for assisting in the management of contaminated inhabited areas in Europe following a radiological emergency, Version 2, in CD EUR 24457 (Commission of the European Communities, Luxembourg, 2011). 